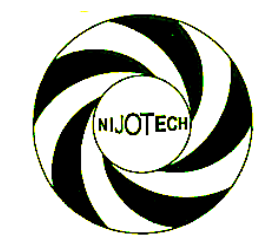

Nigerian Journal of Technology (NIJOTECH)

Vol. 39, No. 1, January 2020, pp. 52 - 62

Copyright@ Faculty of Engineering, University of Nigeria, Nsukka,

Print ISSN: 0331-8443, Electronic ISSN: 2467-8821 www.nijotech.com

http://dx.doi.org/10.4314/njt.v39i1.6

\title{
APPLICATION OF ALTERNATIVE II THEORY TO VIBRATION AND STABILITY ANALYSIS OF THICK RECTANGULAR PLATES (ISOTROPIC AND ORTHOTROPIC)
}

\author{
O. M. Ibearugbulem ${ }^{1, *}$, S. I. Ebirim. ${ }^{2}$, U.C. Anya ${ }^{3}$ and L. O. Ettu ${ }^{4}$ \\ $\mathbf{1 , 2 , 3 , 4}$, DePT OF CIVIL ENGINEERING, FEDERAL UNIVERSITY OF TECHNOLOGY, OWERRI, IMO STATE, NIGERIA \\ E-mail addresses: ${ }^{1}$ ibeowus@gmail.com, ${ }^{2}$ stanley.ebirim@gmail.com, ${ }^{3}$ anyauchukwu@gmail.com
}

\begin{abstract}
This work analysed the free vibration and stability of thick isotropic and orthotropic plates with SSSS and SSFS support conditions by applying the alternative II theory based on polynomial shape function. The total potential energy which was obtained by combining the strain energy and external work was reduced to three governing equations using Ritz method. Polynomial shape function which varies with Poisson's ratio was substituted into the governing equation to obtain the fundamental natural frequency, linear frequency and critical buckling load. The values of frequencies of the first mode and critical loads obtained were compared with those obtained using first order shear deformation theory. For span depth ratio of 10, the fundamental linear frequency for orthotropic SSFS plate corresponding to modulus of elasticity ratios $\left(E_{1} / E_{2}\right)$ of 10,25 and 40 are $0.00156,0.00219$ and $0.00255 \mathrm{~Hz}$. The corresponding values using first order shear deformation theory are $0.00152,0.00212$ and $0.00245 \mathrm{~Hz}$.
\end{abstract}

Keywords: Fundamental natural frequency, SSSS plate, SSFS plate, Ritz method, Orthotropic thick plate, Isotropic thick plate, Stability, Free vibration

\section{INTRODUCTION: ANALYSIS OF PLATES}

The analysis of plate has always been of great interest to researchers. As a result, many plate theories have been developed over the years to provide approximate or exact solutions to plate problems. These theories include classical plate theory (CPT) or Kirchhoff's thin plate theory, firstorder shear deformation plate theory (FSDT), higherorder shear deformation theories (HSDTs), finite element method (FEM) and many more[1].

The CPT is extensively used in static bending, stability and vibrations analyses of thin and thick plates in the part of solid structural mechanics. Even though it is one of the first theories used in the analysis of rectangular plate, it neglected the effect of transverse shear deformation, thereby over estimating natural frequencies. Reissner and Mindlin developed firstorder shear deformation plate theories (FSDTs) bearing in mind the transverse shear and rotary inertia effects by the way of linear variation of inplane translate through the thickness of the plate [2].
The transverse shear strain distribution in FSDT is assumed to be continual through the plate's depth and therefore, a shear correction value is required to justify for the strain energy owing to shear deformation [2]. These shear correction factors, in general are problem dependent. In order to overcome the limitation of CPT and FSDT, many higher-order shear deformation plate theories were developed. They include higher-order shear deformation plate theories [3], refined theories [4], trigonometric shear deformation theory (TSDT) [5] just to mention a few. Some of these higher order theories were evolved from CPT assumptions even though they avoided the linearity of shear deformation line $f(z)$. More so, they are seen to be too rigorous for designers to work with.

Ibearugbulem[6] developed a refined plate theory name alternative I and II which was not based on the CPT assumptions. Though the theory is a first order theory, the shear strain distributed across the depth

* Corresponding author, tel: +2348038357983 
of the plate was assumed not constant. The assumption made for the alternative II theory are:

i. The displacements, $u, v$ and $w$ are small when compared with plate thickness.

ii. The in-plane displacements, $u$ and $v$ are differentiable in $x, y$ and $z$ axes, while the out-of-plane displacement (deflection), $w$ is only differentiable in $x$ and $y$ axes. This means that the first derivative of $w$ with respect to $z$ is zero. Consequently, $\varepsilon z=0$.

iii. The effect of the out-of-plane normal stress on the gross response of the plate is small when compared with other stresses. Thus, it can be neglected. That is $\sigma z=0$.

iv. The CPT component of in-plane strains are not zero. The following relationships hold:

$$
\begin{aligned}
& u=z \frac{d w}{d x}+z \emptyset_{x} \\
& v=z \frac{d w}{d y}+z \emptyset_{y} \\
& \gamma_{x z}=\frac{d u}{d z}+\frac{d w}{d x}=2 \frac{d w}{d x}+\emptyset_{x} \\
& \gamma_{y z}=\frac{d v}{d z}+\frac{d w}{d y}=2 \frac{d w}{d y}+\emptyset_{y}
\end{aligned}
$$

v. The maximum vertical shear stress ( $\tau_{x z m}$ or $\left.\tau_{y z m}\right)$ distributed through the plate thickness is the product of nominal vertical shear stress ( $\tau_{x z}$ or $\tau_{y z}$ ) and shape factor, $G(z)$. That is:

$$
\begin{aligned}
& \tau_{x z m}=\tau_{x z} G(z) \\
& \tau_{x z}=\frac{E}{2(1+\mu)} \gamma_{x z}
\end{aligned}
$$

Therefore,

$$
\tau_{x z m}=\frac{E G(z)}{2(1+\mu)} \gamma_{x z}
$$

Similarly,

$$
\tau_{y z m}=\frac{E G(z)}{2(1+\mu)} \gamma_{y z}
$$

vi. The vertical section that is initially normal to the middle surface of the plate before bending remains straight but no longer normal to the middle surface after bending. That is, $\phi \neq \theta c$. Where $\phi$ is the total vertical rotation of the middle surface and $\theta c$ is the CPT component of the vertical rotation of the middle surface.

Earlier refined plate theory scholars assumed that the vertical shear strain $\left(\gamma_{x z m}\right.$ or $\left.\gamma_{y z m}\right)$ distributed across the thickness of the plate depends on (is function of) shear deformation line, $f(z)$. However, [6] and [7] opined that the vertical shear strain distributed across the depth of the plate is a product of nominal vertical shear strain $\left(\gamma_{x z}\right.$ or $\left.\gamma_{y z}\right)$ and shape factor, $G(z)$. That is; $\gamma_{x z m}=\gamma_{x z} G(z)$ and $\gamma_{y z m}=\gamma_{y z} G(z)$.

Ibearugbulem [6] limited the study to isotropic plates only, out of plane displacement function $w=A_{1} h$ (otherwise called deflection) was not derived from governing Equation but was assumed. Also previous studies on plate tend to be rigorous and time consuming. Thus, this study aims to advance on the shortcomings of previous research works, by applying the alternative II method and the shape factor [8] and [9] to isotropic and orthotropic thick plate.

\section{FORMULATION OF FUNDAMENTAL NATURAL FREQUENCY}

Looking at Figure 1 which represent a deformed plate, the CPT rotation (slope) and shear deformation slope are designated as $\theta_{c}$ and $\theta_{s}$. The total slope of thick plate is the summation of the CPT slope and shear deformation slope. It is designated as $\phi$.

The relationship between depth of plate $z$ and CPT in-plane displacements for classical parts $u_{c}$ and $v_{c}$ are defined mathematically as:

$$
\begin{gathered}
u_{c}=z \theta_{c x}=z \frac{d w}{d x} \\
v_{c}=z \theta_{c y}=z \frac{d w}{d y}
\end{gathered}
$$

Also, the shear deformation displacement can also be written as

$$
\begin{aligned}
& u_{s}=z \theta_{x} \\
& v_{s}=z \theta_{y}
\end{aligned}
$$

Where the subscripts ' $c$ ' and ' $s$ ' denote the classical part and shear deformation part respectively. The deflection (out-of-plane displacement) is denoted as ' $W$. Following Figure 1 for slope, it is taken that addition of the classical and shear deformation parts of the in-plane displacements gives the total in-plane displacements as (adding Equations 9 and 11, and Equations 10 and 12 respectively):

$$
\begin{aligned}
& u=u_{c}+u_{s}=z \frac{d w}{d x}+z \emptyset_{x}=z\left(\frac{d w}{d x}+\emptyset_{x}\right) \\
& v=v_{c}+v_{s}=z \frac{d w}{d y}+z \emptyset_{y}=z\left(\frac{d w}{d y}+\emptyset_{y}\right)
\end{aligned}
$$

Since $\varepsilon_{z}=0$ the remaining engineering strains, which are $\varepsilon_{x}, \varepsilon_{y}, \gamma_{x y}, \gamma_{x z}$ and $\gamma_{y z}$ are defined as follows:

$$
\begin{aligned}
& \varepsilon_{x}=\frac{d u}{d x}=z\left(\frac{d^{2} w}{d x^{2}}+\frac{d \emptyset_{x}}{d x}\right) \\
& \varepsilon_{y}=\frac{d v}{d y}=z\left(\frac{d^{2} w}{d y^{2}}+\frac{d \emptyset_{y}}{d y}\right)
\end{aligned}
$$


Application of Alternative II TheOry to Vibration and Stability ANAlysis of Thick ReCtangular Plates, Ibearugbulem et al.

$$
\begin{aligned}
& \gamma_{x y}=\frac{d u}{d y}+\frac{d v}{d x}=2 z \frac{d^{2} w}{d x d y}+z \frac{d \emptyset_{x}}{d y}+z \frac{d \emptyset_{y}}{d x} \\
& \gamma_{x z}=\frac{d u}{d z}+\frac{d w}{d x}=2 \frac{d w}{d x}+\emptyset_{x} \\
& \gamma_{y z}=\frac{d v}{d z}+\frac{d w}{d y}=2 \frac{d w}{d y}+\emptyset_{y}
\end{aligned}
$$

The stress strain relationship for orthotropic plate are as follows:

$$
\begin{aligned}
& \sigma_{x}=\frac{z E_{0}}{1-\mu_{12} \mu_{21}}\left[e_{11}\left(\frac{d^{2} w}{d x^{2}}+\frac{d \emptyset_{x}}{d x}\right)\right. \\
& \left.+e_{12}\left(\frac{d^{2} w}{d y^{2}}+\frac{d \emptyset_{y}}{d y}\right)\right] \\
& \sigma_{y}=\frac{z E_{0}}{1-\mu_{12} \mu_{21}}\left[e_{22}\left(\frac{d^{2} w}{d y^{2}}+\frac{d \emptyset_{y}}{d y}\right)\right. \\
& \left.+e_{12}\left(\frac{d^{2} w}{d x^{2}}+\frac{d \emptyset_{x}}{d x}\right)\right] \\
& \tau_{x y}=G_{12} \gamma_{x y}=\frac{z E_{0}}{1-\mu_{12} \mu_{21}} e_{33}\left(2 \frac{d^{2} w}{d x d y}+\frac{d \emptyset_{x}}{d y}\right. \\
& \left.+\frac{d \emptyset_{y}}{d x}\right) \\
& \tau_{x z}=G_{13} \gamma_{x z}=\frac{z E_{0}}{1-\mu_{12} \mu_{21}} e_{44}\left(2 \frac{d w}{d x}+\emptyset_{x}\right) \\
& \tau_{y z}=G_{23} \gamma_{y z}=\frac{z E_{0}}{1-\mu_{12} \mu_{21}} e_{55}\left(2 \frac{d w}{d y}+\emptyset_{y}\right)
\end{aligned}
$$

Where:

$$
\begin{gathered}
e_{11}=\frac{E_{1}}{E_{0}} \\
e_{12}=\frac{\mu_{21} \cdot E_{1}}{E_{0}}=\frac{\mu_{12} \cdot E_{2}}{E_{0}} \\
e_{22}=\frac{E_{2}}{E_{0}} \\
e_{33}=G_{12} \frac{\left(1-\mu_{12} \mu_{21}\right)}{E_{0}} \\
e_{44}=G_{13} \frac{\left(1-\mu_{12} \mu_{21}\right)}{E_{0}} \\
e_{55}=G_{23} \frac{\left(1-\mu_{12} \mu_{21}\right)}{E_{0}}
\end{gathered}
$$

$E_{1}$ and $E_{2}$ are elastic moduli of anisotropic material measured along $x$ and $y$ directions.
$\mathrm{G}_{12}, \mathrm{G}_{13}$ and $\mathrm{G}_{23}$ are elastic modulli measured around $x y, x z$ and $y z$ planes.

$E_{0}$ is the reference Elastic modulus. It can be $E_{1}$ or $E_{2}$. The formula for strain energy stored in a continuum is given mathematically as;

$$
U=\frac{1}{2} \iint_{x y}\left[\int_{t / 2}^{t / 2} \sigma \cdot \varepsilon d z\right] d x d y
$$

Where the dot product:

$$
\sigma . \varepsilon=\sigma_{x} \varepsilon_{x}+\sigma_{y} \varepsilon_{y}+\tau_{x y} \gamma_{x y}+\tau_{x z} \gamma_{x z}+\tau_{y z} \gamma_{y z}
$$

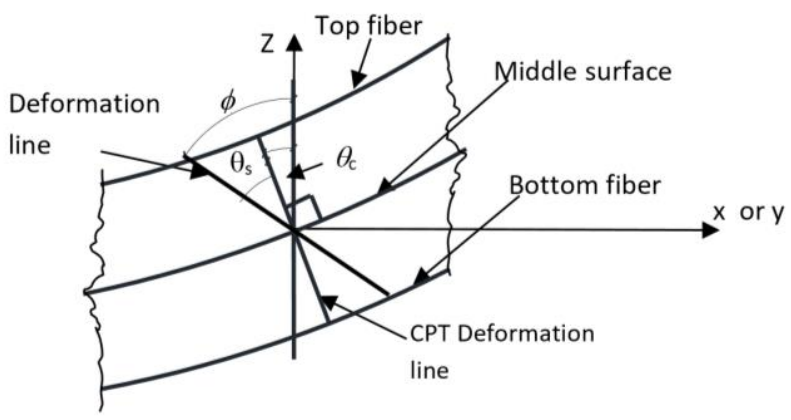

Fig. 1: Plate deformation for alternative II theory

Substituting Equations (15) to (23) into Equation (31) and carrying out the integration with respect to $z$ gives equation (33) at the bottom of this page.

$$
\text { Where: } \quad D_{0}=\frac{E_{0} t^{3}}{12\left\langle 1-\mu_{12} \mu_{21}\right\rangle}
$$

Let:

$$
\begin{aligned}
R & =\frac{x}{a}, 0 \leq R \leq 1 \\
Q & =\frac{y}{b} ; 0 \leq Q \leq 1 \\
P & =\frac{b}{a} \\
\beta & =\frac{a}{t}
\end{aligned}
$$

Where $R$ and $Q$ are non-dimensional coordinates

$$
\begin{aligned}
U=\frac{D_{0}}{2} \int_{0}^{a} \int_{0}^{b}\left\{e_{11}\right. & {\left[\left(\frac{d^{2} w}{d x^{2}}\right)^{2}+2 \frac{d^{2} w}{d x^{2}} \cdot \frac{d \emptyset_{x}}{d x}+\left(\frac{d \emptyset_{x}}{d x}\right)^{2}\right]+2 e_{12}\left[\frac{d^{2} w}{d y^{2}} \cdot \frac{d^{2} w}{d x^{2}}+\frac{d \emptyset_{y}}{d y} \cdot \frac{d^{2} w}{d x^{2}}+\frac{d^{2} w}{d y^{2}} \cdot \frac{d \emptyset_{x}}{d x}+\frac{d \emptyset_{y}}{d y} \cdot \frac{d \emptyset_{x}}{d x}\right] } \\
+e_{22}\left[\left(\frac{d^{2} w}{d y^{2}}\right)^{2}+\right. & \left.\left.2 \frac{d^{2} w}{d y^{2}} \cdot \frac{d \emptyset_{y}}{d y}+\left(\frac{d \emptyset_{y}}{d y}\right)^{2}\right]\right] \\
& +e_{33}\left[4\left(\frac{d^{2} w}{d x d y}\right)^{2}+4 \frac{d \emptyset_{x}}{d y} \frac{d^{2} w}{d x d y}+4 \frac{d \emptyset_{y}}{d x} \frac{d^{2} w}{d x d y}+2 \frac{d \emptyset_{y}}{d x} \frac{d \emptyset_{x}}{d y}+\left(\frac{d \emptyset_{x}}{d y}\right)^{2}+\left(\frac{d \emptyset_{y}}{d x}\right)^{2}\right] \\
& +\frac{12}{t^{2}} e_{44}\left\{\left[4\left(\frac{d w}{d x}\right)^{2}+4 \frac{d w}{d x} \emptyset_{x}+\emptyset_{x}^{2}\right]+\frac{12}{t^{2}} e_{55}\left[4\left(\frac{d w}{d y}\right)^{2}+4 \frac{d w}{d y} \emptyset_{y}+\emptyset_{y}^{2}\right]\right\} d x d y
\end{aligned}
$$

Equation (33) is written in terms of non-dimensional coordinates $R$ and $Q$ as: 
Application of Alternative II TheOry to Vibration and Stability Analysis of Thick ReCtangular Plates, Ibearugbulem et al.

$$
\begin{aligned}
& U=\frac{a b D_{0}}{2 a^{4}} \int_{0}^{1} \int_{0}^{1}\left\{e_{11}\left[\left(\frac{d^{2} w}{d R^{2}}\right)^{2}+2 a \frac{d^{2} w}{d R^{2}} \cdot \frac{d \emptyset_{x}}{d R}+a^{2}\left(\frac{d \emptyset_{x}}{d R}\right)^{2}\right]\right. \\
& +\frac{2}{P^{2}} e_{12}\left[\frac{d^{2} w}{d Q^{2}} \cdot \frac{d^{2} w}{d R^{2}}+b \frac{d \emptyset_{y}}{d Q} \cdot \frac{d^{2} w}{d R^{2}}+a \frac{d^{2} w}{d Q^{2}} \cdot \frac{d \emptyset_{x}}{d R}+a b \frac{d \emptyset_{y}}{d Q} \cdot \frac{d \emptyset_{x}}{d R}\right] \\
& +\frac{1}{P^{4}} e_{22}\left[\left(\frac{d^{2} w}{d Q^{2}}\right)^{2}+2 b \frac{d^{2} w}{d Q^{2}} \cdot \frac{d \emptyset_{y}}{d Q}+b^{2}\left(\frac{d \emptyset_{y}}{d Q}\right)^{2}\right] \\
& +\frac{1}{P^{2}} e_{33}\left[4\left(\frac{d^{2} w}{d R d Q}\right)^{2}+4 a \frac{d \emptyset_{x}}{d Q} \frac{d^{2} w}{d R d Q}+4 b \frac{d \emptyset_{y}}{d R} \frac{d^{2} w}{d R d Q}+2 a b \frac{d \emptyset_{y}}{d R} \frac{d \emptyset_{x}}{d Q}+a^{2}\left(\frac{d \emptyset_{x}}{d Q}\right)^{2}+b^{2}\left(\frac{d \emptyset_{y}}{d R}\right)^{2}\right]
\end{aligned}
$$

The external work of a rectangular plate subject to inertia (dynamic) load and in-plane load [9] is

$$
\begin{gathered}
V=-\frac{a b}{2} \int_{0}^{1} \int_{0}^{1}\left[\rho t w^{2} \omega^{2}+\frac{N_{R}}{a^{2}}\left(\frac{\partial w}{\partial R}\right)^{2}\right] d R d Q=-\frac{a b}{2 a^{4}} \int_{0}^{1} \int_{0}^{1} F_{r} \cdot w^{2} d R d Q \\
\text { Where: } F_{r}=a^{4}\left[\left(\rho t \omega^{2}\right)+\frac{N_{R}}{a^{2}} \frac{\partial^{2}}{\partial R^{2}}\right]
\end{gathered}
$$

Adding Equations (39) and (40) gives the total potential energy functional for thick rectangular plates

$$
\begin{aligned}
& \pi=\frac{a b D_{0}}{2 a^{4}} \int_{0}^{1} \int_{0}^{1}\left\{e_{11}\left[\left(\frac{d^{2} w}{d R^{2}}\right)^{2}+2 a \frac{d^{2} w}{d R^{2}} \cdot \frac{d \emptyset_{x}}{d R}+a^{2}\left(\frac{d \emptyset_{x}}{d R}\right)^{2}\right]\right. \\
& +\frac{2}{P^{2}} e_{12}\left[\frac{d^{2} w}{d Q^{2}} \cdot \frac{d^{2} w}{d R^{2}}+b \frac{d \emptyset_{y}}{d Q} \cdot \frac{d^{2} w}{d R^{2}}+a \frac{d^{2} w}{d Q^{2}} \cdot \frac{d \emptyset_{x}}{d R}+a b \frac{d \emptyset_{y}}{d Q} \cdot \frac{d \emptyset_{x}}{d R}\right] \\
& +\frac{1}{P^{4}} e_{22}\left[\left(\frac{d^{2} w}{d Q^{2}}\right)^{2}+2 b \frac{d^{2} w}{d Q^{2}} \cdot \frac{d \emptyset_{y}}{d Q}+b^{2}\left(\frac{d \emptyset_{y}}{d Q}\right)^{2}\right] \\
& +\frac{1}{P^{2}} e_{33}\left[4\left(\frac{d^{2} w}{d R d Q}\right)^{2}+4 a \frac{d \emptyset_{x}}{d Q} \frac{d^{2} w}{d R d Q}+4 b \frac{d \emptyset_{y}}{d R} \frac{d^{2} w}{d R d Q}+2 a b \frac{d \emptyset_{y}}{d R} \frac{d \emptyset_{x}}{d Q}+a^{2}\left(\frac{d \emptyset_{x}}{d Q}\right)^{2}+b^{2}\left(\frac{d \emptyset_{y}}{d R}\right)^{2}\right] \\
& +12 \beta^{2} e_{44}\left[4\left(\frac{d w}{d R}\right)^{2}+4 a \frac{d w}{d R} \emptyset_{x}+a^{2} \emptyset_{x}{ }^{2}\right]+\frac{12}{P^{2}} \beta^{2} e_{55}\left[4\left(\frac{d w}{d Q}\right)^{2}+4 b \frac{d w}{d Q} \emptyset_{y}+b^{2} \emptyset_{y}{ }^{2}\right]
\end{aligned}
$$

Apply Ritz method to Equation (42). That is, minimizing Equation (42) in turn with respect to $w$, $\phi_{x}$ and $\phi_{y}$ respectively gives

$$
\begin{aligned}
& \frac{\partial \pi}{\partial w}=\frac{a b D_{0}}{a^{4}} \int_{0}^{1} \int_{0}^{1}\left\{e_{11}\left[\frac{d^{4} w}{d R^{4}}+a \frac{d^{3} \emptyset_{x}}{d R^{3}}\right]+\frac{1}{P^{2}} e_{12}\left[2 \frac{d^{4} w}{d Q^{2} d R^{2}}+a \frac{d^{3} \emptyset_{x}}{d Q^{2} d R}+b \frac{d^{3} \emptyset_{y}}{d Q d R^{2}}\right]\right. \\
& +\frac{1}{P^{4}} e_{22}\left[\frac{d^{4} w}{d Q^{4}}+b \frac{d^{3} \emptyset_{y}}{d Q^{3}}\right]+\frac{1}{P^{2}} e_{33}\left[4 \frac{d^{4} w}{d R^{2} d Q^{2}}+2 a \frac{d^{3} \emptyset_{x}}{d R d Q^{2}}+2 b \frac{d^{3} \emptyset_{y}}{d R^{2} d Q}\right] \\
& \left.+12 \beta^{2} e_{44}\left[4 \frac{d^{2} w}{d R^{2}}+2 a \frac{d \emptyset_{x}}{d R}\right]+\frac{12}{P^{2}} \beta^{2} e_{55}\left[4 \frac{d^{2} w}{d Q^{2}}+2 b \frac{d \emptyset_{y}}{d Q}\right]-F_{r} \cdot w\right\} d R d Q=0 \\
& \frac{\partial \pi}{\partial \varphi_{x}}=\frac{a b D_{0}}{a^{3}} \int_{0}^{1} \int_{0}^{1}\left\{e_{11}\left[\frac{d^{3} w}{d R^{3}}+a \frac{d^{2} \emptyset_{x}}{d R^{2}}\right]+\frac{1}{P^{2}} e_{12}\left[\frac{d^{3} w}{d Q^{2} d R}+b \frac{d^{2} \emptyset_{y}}{d Q d R}\right]\right. \\
& \left.+\frac{1}{P^{2}} e_{33}\left[2 \frac{d^{3} w}{d R d Q^{2}}+b \frac{d^{2} \emptyset_{y}}{d R d Q}+a \frac{d^{2} \emptyset_{x}}{d Q^{2}}\right]+12 \beta^{2} e_{44}\left[2 \frac{d w}{d R}+a \emptyset_{x}\right]\right\} d R d Q=0 \\
& \frac{\partial \pi}{\partial \varphi_{y}}=\frac{a b D_{0}}{a^{3}} \int_{0}^{1} \int_{0}^{1}\left\{\frac{1}{P} e_{12}\left[\frac{d^{3} w}{d Q d R^{2}}+a \frac{d^{2} \emptyset_{x}}{d Q d R}\right]+\frac{1}{P^{3}} e_{22}\left[+\frac{d^{3} w}{d Q^{3}}+b \frac{d^{2} \emptyset_{y}}{d Q^{2}}\right]\right. \\
& \left.+\frac{1}{P} e_{33}\left[2 \frac{d^{3} w}{d^{2} R d Q}+a \frac{d^{2} \emptyset_{x}}{d R d Q}+b \frac{d^{2} \emptyset_{y}}{d R^{2}}\right]+\frac{12}{P} \beta^{2} e_{55}\left[2 \frac{d w}{d Q}+b \emptyset_{y}\right]\right\} d R d Q=0
\end{aligned}
$$

Note: Equation (45), (46) and (47) are called governing equation for rectangular plate 
Application of Alternative II TheOry to Vibration and Stability Analysis of Thick ReCtangular Plates, Ibearugbulem et al.

From Equation 46, the following conditions must hold:

$$
\begin{aligned}
& \left(\frac{d^{3} w}{d R^{3}}+a \frac{d^{2} \emptyset_{x}}{d R^{2}}\right)=0 \\
& \left(\frac{d^{3} w}{d Q^{2} d R}+b \frac{d^{2} \emptyset_{y}}{d Q d R}\right)=0 \\
& \left(2 \frac{d^{3} w}{d R d Q^{2}}+b \frac{d^{2} \emptyset_{y}}{d R d Q}+a \frac{d^{2} \emptyset_{x}}{d Q^{2}}\right)=0 \\
& \left(2 \frac{d w}{d R}+a \emptyset_{x}\right)=0
\end{aligned}
$$

From Equation 47, the following conditions must hold:

$$
\begin{aligned}
& \left(\frac{d^{3} w}{d Q d R^{2}}+a \frac{d^{2} \emptyset_{x}}{d Q d R}\right)=0 \\
& \left(+\frac{d^{3} w}{d Q^{3}}+b \frac{d^{2} \emptyset_{y}}{d Q^{2}}\right)=0 \\
& \left(2 \frac{d^{3} w}{d^{2} R d Q}+a \frac{d^{2} \emptyset_{x}}{d R d Q}+b \frac{d^{2} \emptyset_{y}}{d R^{2}}\right)=0 \\
& \iint_{R Q}\left[e_{11}\left(\mathrm{w}_{\mathrm{Q}} \frac{d^{4} \mathrm{w}_{\mathrm{R}}}{d R^{4}}+a \emptyset_{\mathrm{RQ}} \frac{d^{3} \emptyset_{\mathrm{RR}}}{d R^{3}}\right)+\frac{1}{P^{2}} e_{12}\left(2 \mathrm{w}_{\mathrm{Q}} \frac{d^{4} \mathrm{w}_{\mathrm{R}}}{d Q^{2} d R^{2}}+a \emptyset_{\mathrm{RQ}} \frac{d^{3} \emptyset_{\mathrm{RR}}}{d Q^{2} d R}+b \emptyset_{\mathrm{QR}} \frac{d^{3} \emptyset_{\mathrm{QQ}}}{d Q d R^{2}}\right)\right. \\
& +\frac{1}{P^{4}} e_{22}\left(\mathrm{w}_{\mathrm{R}} \frac{d^{4} \mathrm{w}_{\mathrm{Q}}}{d Q^{4}}+b \emptyset_{\mathrm{QR}} \frac{d^{3} \emptyset_{\mathrm{QQ}}}{d Q^{3}}\right)+\frac{1}{P^{2}} e_{33}\left(4 \mathrm{w}_{\mathrm{Q}} \frac{d^{4} \mathrm{w}_{\mathrm{R}}}{d R^{2} d Q^{2}}+2 a \emptyset_{\mathrm{RQ}} \frac{d^{3} \emptyset_{\mathrm{RR}}}{d R d Q^{2}}+2 b \emptyset_{\mathrm{QR}} \frac{d^{3} \emptyset_{\mathrm{QQ}}}{d R^{2} d Q}\right) \\
& +12 \beta^{2} e_{44}\left(4 \mathrm{w}_{\mathrm{Q}} \frac{d^{2} \mathrm{w}_{\mathrm{R}}}{d R^{2}}+2 a \emptyset_{\mathrm{RQ}} \frac{d \emptyset_{\mathrm{RR}}}{d R}\right)+\frac{12}{P^{2}} \beta^{2} e_{55}\left(4 \mathrm{w}_{\mathrm{R}} \frac{d^{2} \mathrm{w}_{\mathrm{Q}}}{d Q^{2}}+2 b \emptyset_{\mathrm{QR}} \frac{d \emptyset_{\mathrm{QQ}}}{d Q}\right) \\
& \left.-\frac{F_{r}}{D_{0}} \cdot \mathrm{w}_{\mathrm{R}} \cdot \mathrm{w}_{\mathrm{Q}}\right] d R d Q=0 \\
& \mathrm{w}=\mathrm{w}_{\mathrm{R}} \cdot \mathrm{w}_{\mathrm{Q}} \\
& \emptyset_{\mathrm{R}}=\emptyset_{\mathrm{RR}} \cdot \emptyset_{\mathrm{RQ}} \\
& \emptyset_{\mathrm{Q}}=\emptyset_{\mathrm{QR}} \cdot \varnothing
\end{aligned}
$$

Solving Equation (48) to (51) and (52) to (55) gives:

$$
\begin{aligned}
& \emptyset_{x}=\frac{B_{2}}{a} \frac{d w}{d R} \\
& \emptyset_{y}=\frac{B_{3}}{b} \frac{d w}{d Q}
\end{aligned}
$$

Where $B_{2}$ and $B_{3}$ are yet to be determined constants.

By employing split displacements, the following displacements were defined as:

Let $F_{r}=-\mathrm{n}_{1} F_{r}-\mathrm{n}_{2} F_{r}$ and collect like terms in Equation (61) to obtain

$$
\begin{aligned}
\iint_{R Q}\left[\left(e_{11} \mathrm{w}_{\mathrm{Q}} \frac{d^{4} \mathrm{w}_{\mathrm{R}}}{d R^{4}}\right.\right. & \left.+n_{1} \cdot \frac{F_{r}}{D_{0}} \cdot \mathrm{w}_{\mathrm{R}} \cdot \mathrm{w}_{\mathrm{Q}}\right)+\left(\frac{1}{P^{4}} e_{22} \mathrm{w}_{\mathrm{R}} \frac{d^{4} \mathrm{w}_{\mathrm{Q}}}{d Q^{4}}+n_{2} \cdot \frac{F_{r}}{D_{0}} \cdot \mathrm{w}_{\mathrm{R}} \cdot \mathrm{w}_{\mathrm{Q}}\right) \\
& +\frac{1}{P^{2}} e_{12}\left(2 \mathrm{w}_{\mathrm{Q}} \frac{d^{4} \mathrm{w}_{\mathrm{R}}}{d Q^{2} d R^{2}}+a \emptyset_{\mathrm{RQ}} \frac{d^{3} \emptyset_{\mathrm{RR}}}{d Q^{2} d R}+b \emptyset_{\mathrm{QR}} \frac{d^{3} \emptyset_{\mathrm{QQ}}}{d Q d R^{2}}\right) \\
& +\frac{1}{P^{2}} e_{33}\left(4 \mathrm{w}_{\mathrm{Q}} \frac{d^{4} \mathrm{w}_{\mathrm{R}}}{d R^{2} d Q^{2}}+2 a \emptyset_{\mathrm{RQ}} \frac{d^{3} \emptyset_{\mathrm{RR}}}{d R d Q^{2}}+2 b \emptyset_{\mathrm{QR}} \frac{d^{3} \emptyset_{\mathrm{QQ}}}{d R^{2} d Q}\right) \\
& +\left(e_{11} a \emptyset_{\mathrm{RQ}} \frac{d^{3} \emptyset_{\mathrm{RR}}}{d R^{3}}+\frac{1}{P^{4}} e_{22} b \emptyset_{\mathrm{QR}} \frac{d^{3} \emptyset_{\mathrm{QQ}}}{d Q^{3}}\right)+12 \beta^{2} e_{44}\left(4 \mathrm{w}_{\mathrm{Q}} \frac{d^{2} \mathrm{w}_{\mathrm{R}}}{d R^{2}}+2 a \emptyset_{\mathrm{RQ}} \frac{d \emptyset_{\mathrm{RR}}}{d R}\right) \\
& \left.+\frac{12}{P^{2}} \beta^{2} e_{55}\left(4 \mathrm{w}_{\mathrm{R}} \frac{d^{2} \mathrm{w}_{\mathrm{Q}}}{d Q^{2}}+2 b \emptyset_{\mathrm{QR}} \frac{d \emptyset_{\mathrm{QQ}}}{d Q}\right)\right] d R d Q=0
\end{aligned}
$$

One of the conditions for Equation 62 to be valid is for the following to be zeros:

$$
\begin{aligned}
& \iint_{R Q}\left(e_{11} \mathrm{w}_{\mathrm{Q}} \frac{d^{4} \mathrm{w}_{\mathrm{R}}}{d R^{4}}+n_{1} \cdot \frac{F_{r}}{D_{0}} \cdot \mathrm{w}_{\mathrm{R}} \cdot \mathrm{w}_{\mathrm{Q}}\right) d R d Q=0 \\
& \iint_{R Q}\left(\frac{1}{P^{4}} e_{22} \mathrm{w}_{\mathrm{R}} \frac{d^{4} \mathrm{w}_{Q}}{d Q^{4}}+n_{2} \cdot \frac{F_{r}}{D_{0}} \cdot \mathrm{w}_{\mathrm{R}} \cdot \mathrm{w}_{\mathrm{Q}}\right) d R d Q=0 \\
& \iint_{R Q} \frac{1}{P^{2}} e_{12}\left(2 w_{Q} \frac{d^{4} w_{R}}{d Q^{2} d R^{2}}+a \emptyset_{R Q} \frac{d^{3} \emptyset_{R R}}{d Q^{2} d R}+b \emptyset_{Q R} \frac{d^{3} \emptyset_{Q Q}}{d Q d R^{2}}\right) d R d Q=0 \\
& \iint_{R Q} \frac{1}{P^{2}} e_{33}\left(4 w_{Q} \frac{d^{4} w_{R}}{d R^{2} d Q^{2}}+2 a \emptyset_{R Q} \frac{d^{3} \emptyset_{R R}}{d R d Q^{2}}+2 b \emptyset_{Q R} \frac{d^{3} \emptyset_{Q Q}}{d R^{2} d Q}\right) d R d Q=0 \\
& \iint_{R Q}\left(e_{11} a \varphi_{R Q} \frac{d^{3} \emptyset_{R R}}{d R^{3}}+\frac{1}{P^{4}} e_{22} b \emptyset_{Q R} \frac{d^{3} \emptyset_{Q Q}}{d Q^{3}}\right) d R d Q=0
\end{aligned}
$$


Application of Alternative II Theory to Vibration ANd Stability ANalysis Of Thick ReCtANGUlar Plates, Ibearugbulem et al.

$$
\begin{aligned}
& \iint_{R Q} 12 \beta^{2} e_{44}\left(4 w_{Q} \frac{d^{2} w_{R}}{d R^{2}}+2 a \emptyset_{R Q} \frac{d \emptyset_{R R}}{d R}\right) d R d Q=0 \\
& \iint_{R Q} \frac{12}{P^{2}} \beta^{2} e_{55}\left(4 w_{R} \frac{d^{2} w_{Q}}{d Q^{2}}+2 b \emptyset_{Q R} \frac{d \emptyset_{Q Q}}{d Q}\right) d R d Q=0
\end{aligned}
$$

From Equation (63)

$$
\frac{d^{4} w_{R}}{d R^{4}}+\left(\frac{n_{1} \cdot F_{r} \cdot w_{Q}}{D_{0} e_{11} w_{Q}}\right) w_{R}=\frac{d^{4} w_{R}}{d R^{4}}+k_{1}{ }^{4} w_{R}=0
$$

From Equation (64)

$$
\frac{d^{4} w_{Q}}{d Q^{4}}+\left(\frac{n_{2} \cdot F_{r} \cdot w_{R}}{D_{0} P^{4} e_{22} w_{R}}\right) w_{Q}=\frac{d^{4} w_{Q}}{d Q^{4}}+k_{2}^{4} w_{Q}=0
$$

Prepared and approximate solutions to Equations (70) and (71) in polynomial forms are:

$$
w_{R}=a_{0}+a_{1} R+a_{2} R^{2}+a_{3} R^{3}+a_{4} R^{4}=\left[\begin{array}{lll}
1 & R & R^{2} R^{3} R^{4}
\end{array}\right]\left[\begin{array}{l}
a_{1} \\
a_{2} \\
a_{3} \\
a_{4}
\end{array}\right]=A_{R} h_{R}
$$

Substituting Equations (72) and (73) into Equation (58) gives:

$$
\begin{aligned}
& \mathrm{w}=\mathrm{w}_{\mathrm{R}} \cdot \mathrm{w}_{\mathrm{Q}}=\left(A_{R} \cdot A_{Q}\right)\left(h_{R} \cdot h_{Q}\right)=A_{1} h \\
& \text { Where: } A_{1}=A_{R} \cdot A_{Q} \text { and } h=h_{R} \cdot h_{Q}
\end{aligned}
$$

Substituting Equation (74) into Equations (56) and (57) gives:

$$
\begin{aligned}
& \emptyset_{x}=\frac{B_{2}}{a} \frac{d A_{1} h}{d R}=\frac{A_{2}}{a} \frac{d h}{d R} \\
& \emptyset_{y}=\frac{B_{3}}{b} \frac{d A_{1} h}{d Q}=\frac{A_{3}}{b} \frac{d h}{d Q} \\
& A_{2}=A_{1} B_{2} \\
& A_{3}=A_{1} B_{3}
\end{aligned}
$$

Substituting Equations (74), (76) and (77) into Equation (42) gives:

$$
\begin{gathered}
\pi=\frac{a b}{2 a^{4}} \int_{0}^{1} \int_{0}^{1}\left\{e_{11}\left[{A_{1}}^{2}+2 A_{1} A_{2}+{A_{2}}^{2}\right]\left(\frac{d^{2} h}{d R^{2}}\right)^{2}+\frac{2}{P^{2}} e_{12}\left[A_{1}{ }^{2}+A_{1} A_{2}+A_{1} A_{3}+A_{2} A_{3}\right]\left(\frac{d^{2} h}{d R d Q}\right)^{2}\right. \\
+\frac{1}{P^{4}} e_{22}\left[A_{1}{ }^{2}+2 A_{1} A_{3}+{A_{3}}^{2}\right]\left(\frac{d^{2} h}{d Q^{2}}\right)^{2}+\frac{1}{P^{2}} e_{33}\left[4{A_{1}}^{2}+4 A_{1} A_{2}+4 A_{1} A_{3}+2 A_{2} A_{3}+{A_{2}}^{2}+A_{3}{ }^{2}\right]\left(\frac{d^{2} h}{d R d Q}\right)^{2} \\
\left.+12 \beta^{2} e_{44}\left[4{A_{1}}^{2}+4 A_{1} A_{2}+{A_{2}}^{2}\right]\left(\frac{d h}{d R}\right)^{2}+\frac{12}{P^{2}} \beta^{2} e_{55}\left[4{A_{1}}^{2}+4 A_{1} A_{3}+A_{3}{ }^{2}\right]\left(\frac{d h}{d Q}\right)^{2}-A_{1}{ }^{2} \frac{F_{r}}{D_{0}} \cdot h^{2}\right\} d R d Q
\end{gathered}
$$

Minimizing Equation (80) in turn with respect to $A_{1}, A_{2}$ and $A_{3}$.i.e. Ritz method

$$
\frac{d \pi}{d A_{1}}=\int_{0}^{1} \int_{0}^{1}\left\{e_{11}\left[A_{1}+A_{2}\right]\left(\frac{d^{2} h}{d R^{2}}\right)^{2}+\frac{1}{P^{2}} e_{12}\left[2 A_{1}+A_{2}+A_{3}\right]\left(\frac{d^{2} h}{d R d Q}\right)^{2}+\frac{1}{P^{4}} e_{22}\left[A_{1}+A_{3}\right]\left(\frac{d^{2} h}{d Q^{2}}\right)^{2}\right.
$$


Application of Alternative II Theory to Vibration and Stability Analysis of Thick Rectangular Plates, lbearugbulem et al.

$$
\begin{aligned}
& +\frac{2}{P^{2}} e_{33}\left[2 A_{1}+A_{2}+A_{3}\right]\left(\frac{d^{2} h}{d R d Q}\right)^{2}+24 \beta^{2} e_{44}\left[2 A_{1}+A_{2}\right]\left(\frac{d h}{d R}\right)^{2}+\frac{24}{P^{2}} \beta^{2} e_{55}\left[2 A_{1}+A_{3}\right]\left(\frac{d h}{d Q}\right)^{2} \\
& \left.-\frac{a^{4}}{D_{0}}\left[\left(\rho t \omega^{2}\right) A_{1} \cdot h^{2}+\frac{N_{R}}{a^{2}} A_{1} \cdot\left(\frac{d h}{d R}\right)^{2}\right]\right\} d R d Q=0 \\
& \frac{d \pi}{d A_{2}}=\int_{0}^{1} \int_{0}^{1}\left\{e_{11}\left[A_{1}+A_{2}\right]\left(\frac{d^{2} h}{d R^{2}}\right)^{2}+\frac{1}{P^{2}} e_{12}\left[A_{1}+A_{3}\right]\left(\frac{d^{2} h}{d R d Q}\right)^{2}+\frac{1}{P^{2}} e_{33}\left[2 A_{1}+A_{3}+A_{2}\right]\left(\frac{d^{2} h}{d R d Q}\right)^{2}\right. \\
& \left.+12 \beta^{2} e_{44}\left[2 A_{1}+A_{2}\right]\left(\frac{d h}{d R}\right)^{2}\right\} d R d Q=0 \\
& \frac{d \pi}{d A_{3}}=\int_{0}^{1} \int_{0}^{1}\left\{\frac{1}{P^{2}} e_{12}\left[A_{1}+A_{2}\right]\left(\frac{d^{2} h}{d R d Q}\right)^{2}+\frac{1}{P^{4}} e_{22}\left[A_{1}+A_{3}\right]\left(\frac{d^{2} h}{d Q^{2}}\right)^{2}+\frac{1}{P^{2}} e_{33}\left[2 A_{1}+A_{2}+A_{3}\right]\left(\frac{d^{2} h}{d R d Q}\right)^{2}\right. \\
& \left.+\frac{12}{P^{2}} \beta^{2} e_{55}\left[2 A_{1}+A_{3}\right]\left(\frac{d h}{d Q}\right)^{2}\right\} d R d Q
\end{aligned}
$$

Rearranging and summarizing Equations (81), (82) and (83) in symbolized forms gives:

Where;

$$
\begin{aligned}
& r_{11} A_{1}+r_{12} A_{2}+r_{13} A_{3}=\frac{a^{4}}{D_{0}}\left[\left(\rho t \omega^{2}\right) A_{1} \cdot k_{\lambda}+\frac{N_{R}}{a^{2}} A_{1} \cdot k_{N R}\right] \\
& r_{22} A_{2}+r_{23} A_{3}=-r_{21} A_{1} \\
& r_{32} A_{2}+r_{33} A_{3}=-r_{31} A_{1}
\end{aligned}
$$

$r_{11}=e_{11} k_{R}+\frac{2}{P^{2}} e_{12} k_{R Q}+\frac{1}{P^{4}} e_{22} k_{Q}+\frac{4}{P^{2}} e_{33} k_{R Q}+48 \beta^{2} e_{44} k_{N R}+\frac{48}{P^{2}} \beta^{2} e_{55} k_{N Q}$

$r_{12}=e_{11} k_{R}+\frac{1}{P^{2}} e_{12} k_{R Q}+\frac{2}{P^{2}} e_{33} k_{R Q}+24 \beta^{2} e_{44} k_{N R} ; \quad r_{22}=e_{11} k_{R}+\frac{1}{P^{2}} e_{33} k_{R Q}+12 \beta^{2} e_{44} k_{N R}$

$r_{13}=\frac{1}{P^{2}} e_{12} k_{R Q}+\frac{1}{P^{4}} e_{22} k_{Q}+\frac{2}{P^{2}} e_{33} k_{R Q}+\frac{24}{P^{2}} \beta^{2} P^{2} e_{55} k_{N Q} ; r_{23}=\frac{1}{P^{2}} e_{12} k_{R Q}+\frac{1}{P^{2}} e_{33} k_{R Q}$

$r_{33}=\frac{1}{P^{4}} e_{22} k_{Q}+\frac{1}{P^{2}} e_{33} k_{R Q}+\frac{12}{P^{2}} \beta^{2} e_{55} k_{N Q} ; \quad r_{21}=r_{12} ; r_{31}=r_{13} ; \quad r_{32}=r_{23}$

$k_{R}=\iint_{00}^{11}\left(\frac{d^{2} h}{d R^{2}}\right)^{2} d R d Q ; \quad k_{R Q}=\iint_{00}^{11}\left(\frac{d^{2} h}{d R d Q}\right)^{2} d R d Q ; \quad k_{Q}=\iint_{00}^{11}\left(\frac{d^{2} h}{d Q^{2}}\right)^{2} d R d Q$

$k_{N R}=\iint_{00}^{11}\left(\frac{d h}{d R}\right)^{2} d R d Q: \quad k_{N R}=\iint_{00}^{11}\left(\frac{d h}{d Q}\right)^{2} d R d Q: \quad k_{\lambda}=\iint_{00}^{11} h^{2} d R d Q ; k_{q}=\iint_{00}^{11} h d R d Q$

Solving Equations (85) and (86) gives:

$A_{2}=T_{2} A_{1}$

$A_{3}=T_{3} A_{1}$

$$
\begin{gathered}
\text { where } T_{2}=\frac{\left(r_{23} r_{31}-r_{21} r_{33}\right)}{\left(r_{22} r_{33}-r_{23} r_{23}\right)} ; \quad T_{3} \\
=\frac{\left(r_{23} r_{21}-r_{22} r_{31}\right)}{\left(r_{22} r_{33}-r_{23} r_{23}\right)}
\end{gathered}
$$

Substituting Equations (87) and (88) into Equation (84) gives:

$$
r_{11}+r_{12} T_{2}+r_{13} T_{3}=\frac{a^{4}}{D_{0}}\left[\left(\rho t \omega^{2}\right) \cdot k_{\lambda}+\frac{N_{R}}{a^{2}} \cdot k_{N R}\right]
$$

Rearranging Equation (89) for stability and free vibration analyses gives:

$$
\begin{gathered}
\frac{N_{R} a^{2}}{D_{0}}=\frac{r_{11}+r_{12} T_{2}+r_{13} T_{3}}{k_{N R}} \\
\frac{\rho t \omega^{2} a^{4}}{D_{0}}=\frac{r_{11}+r_{12} T_{2}+r_{13} T_{3}}{k_{\lambda}}
\end{gathered}
$$

$$
\omega\left(a^{2} \sqrt{\frac{\rho t}{D_{0}}}\right)=\sqrt{\frac{r_{11}+r_{12} T_{2}+r_{13} T_{3}}{k_{\lambda}}}=\omega_{0}
$$

Let the non-dimensional form of the fundamental natural frequency be defined as:

$$
\bar{\omega}=\omega t \sqrt{\frac{\rho}{G}}
$$

Substituting Equation (92) into Equation (93) gives:

$$
\bar{\omega}=\frac{\omega_{0}}{a^{2}} \times \sqrt{\frac{D_{0}}{\rho t}} \times t \sqrt{\frac{\rho}{G}}=\frac{\omega_{0}}{a^{2}} \times \sqrt{\frac{D_{0} t}{G}}
$$

Equation (34) can be rewritten as:

$$
D_{0}=\frac{E_{0}}{2\left\langle 1+\sqrt{\mu_{12} \mu_{21}}\right\rangle} \times \frac{t^{3}}{6\left\langle 1-\sqrt{\mu_{12} \mu_{21}}\right\rangle}
$$

For isotropic case, the shear modulus is defined as:

$$
G=\frac{E_{0}}{2\left\langle 1+\sqrt{\mu_{12} \mu_{21}}\right\rangle}
$$

Substituting Equation (96) into Equation (95) gives: 
Application of Alternative II TheOry to Vibration and Stability Analysis of Thick ReCtangular Plates, Ibearugbulem et al.

$$
D_{0}=G \times \frac{t^{3}}{6\left\langle 1-\sqrt{\mu_{12} \mu_{21}}\right\rangle}
$$

Substituting Equations (97) and (95) into Equation (94) and simplifying gives:

$$
\begin{array}{r}
\bar{\omega}=\frac{\omega_{0}}{a^{2}} \times \sqrt{\frac{G \times \frac{t^{3}}{6\left\langle 1-\sqrt{\mu_{12} \mu_{21}}\right\rangle} \times t}{G}} \\
=\frac{\omega_{0}}{a^{2}} \times \sqrt{\frac{t^{4}}{6\left\langle 1-\sqrt{\mu_{12} \mu_{21}}\right\rangle}} \\
\bar{\omega}=\frac{1}{(a / t)^{2}} \times \sqrt{\frac{r_{11}+r_{12} T_{2}+r_{13} T_{3}}{6\left\langle 1-\sqrt{\mu_{12} \mu_{21}} \times k_{\lambda}\right.}}
\end{array}
$$

The natural frequency, $\omega$ of a plate is related to vibration period, $T$ (or linear frequency, $\lambda$ ) by the following relationship [10]:

$$
\omega=2 \pi \cdot \frac{1}{T}=2 \pi \cdot \lambda
$$

Whereas the unit of natural frequency of the plate is $\mathrm{rad} / \mathrm{s}$, the unit of linear frequency is cycle/s (or Hertz).

Rearranging Equation (92) gives:

$$
\omega a^{2}=\sqrt{\frac{r_{11}+r_{12} T_{2}+r_{13} T_{3}}{k_{\lambda}}} \times \sqrt{\frac{D_{0}}{\rho t}}
$$

Substituting Equation (38) into Equation (100) and rearranging gives

$$
\omega=\frac{1}{a \beta} \sqrt{\frac{E_{0} \cdot\left(r_{11}+r_{12} T_{2}+r_{13} T_{3}\right)}{12\left\langle 1-\mu_{12} \mu_{21}\right\rangle \cdot \rho \cdot k_{\lambda}}}
$$

Converting the natural frequency of the plate to linear frequency, $\lambda$ gives

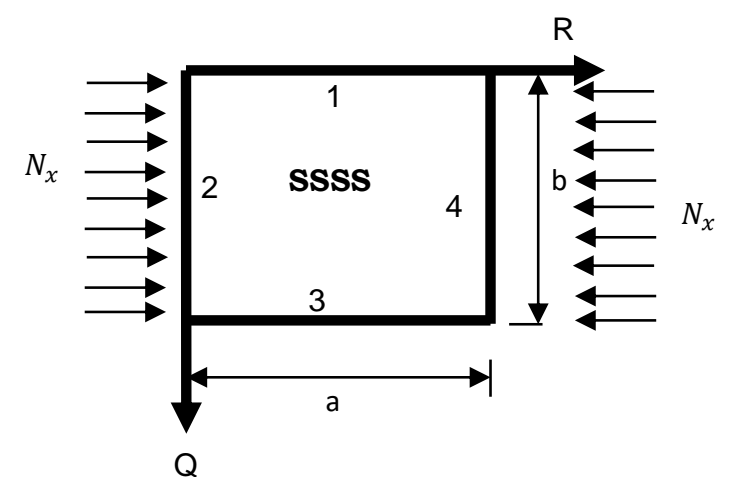

Fig. 3: Numbering Style SSSS Plate

$$
\lambda=\frac{1}{2 \pi a \beta} \sqrt{\frac{E_{0} \cdot\left(r_{11}+r_{12} T_{2}+r_{13} T_{3}\right)}{12\left\langle 1-\mu_{12} \mu_{21}\right\rangle \cdot \rho \cdot k_{\lambda}}}
$$

For stability, substituting Equation (38) into Equation (90) gives

$$
\frac{N_{R} a^{2}}{\left(\frac{E_{0} t^{3}}{12\left\langle 1-\mu_{12} \mu_{21}\right\rangle}\right)}=\frac{r_{11}+r_{12} T_{2}+r_{13} T_{3}}{k_{N R}}
$$

Which on simplifying gives:

$$
\frac{N_{R} a^{2}}{E_{0} t^{3}}=\frac{1}{12\left\langle 1-\mu_{12} \mu_{21}\right\rangle} \cdot\left(\frac{r_{11}+r_{12} T_{2}+r_{13} T_{3}}{k_{N R}}\right)
$$

Equations (102) is strictly for linear frequency for thin and thick isotropic rectangular plate while Equations (101) and (103) can be used for natural frequency and stability analyses of isotropic and orthotropic rectangular thin and thick plate.

\section{NUMERICAL EXAMPLE}

It is desired to determine the critical buckling loads, fundamental natural and linear frequencies for SSSS and SSFS rectangular thick plates. The values of parameters used are [11]. For stability of orthotropic plate (SSSS and SSFS), $E_{1} / E_{2}=10,25$ and 40 , $G_{12} / E_{2}=0.5, G_{13} / E_{2}=0.5, G_{23} / E_{2}=0.2, v_{12}=$ 0.25 . The diagrams of SSSS and SSFS rectangular plates loaded with in-plane load along $x$ direction are shown on Figures 2 and 3.

Table 1: $K$ value for SSSS and SSFS plates

\begin{tabular}{ccc}
\hline Parameters & SSSS & SSFS \\
\hline$K_{R}$ & 0.23619 & $\left(0.3333 S_{2}{ }^{2}+0.4 S_{2} T_{2}+0.3333 S_{2}+0.1429 T_{2}{ }^{2}+0.25 T_{2}+0.11\right) \times 4.8$ \\
\hline$K_{R Q}$ & 0.23592 & $\left(S_{2}{ }^{2}+2 S T_{2}+2 S_{2}+1.8 T_{2}{ }^{2}+4 T_{2}+2.286\right) \times 0.4857$ \\
\hline$K_{Q}$ & 0.23619 & $\left(12 T_{2}{ }^{2}+36 T_{2}+28.80\right) \times 0.0492$ \\
\hline$K_{N R}$ & 0.02390 & $\left(0.3333 S_{2}{ }^{2}+0.4 S T_{2}+0.3333 S_{2}+0.1429 T_{2}{ }^{2}+0.25 T_{2}+0.11\right) \times 0.4857$ \\
\hline$K_{N Q}$ & 0.02390 & $\left(S_{2}{ }^{2}+2 S T_{2}+2 S_{2}+1.8 T_{2}{ }^{2}+4 T_{2}+2.286\right) \times 0.0492$ \\
\hline
\end{tabular}


Application of Alternative II Theory to Vibration and Stability ANalysis Of Thick Rectangular Plates, lbearugbulem et al.

\begin{tabular}{clc}
\hline Parameters & SSSS & SSFS \\
\hline$K_{\lambda}$ & 0.00242 & $\left(0.3333 S_{2}{ }^{2}+0.4 S_{2} T_{2}+0.3333 S_{2}+0.1429 T_{2}{ }^{2}+0.25 T_{2}+0.11\right) \times 0.0492$ \\
\hline$K_{q}$ & 0.0400 & $\left(0.5 S_{S}+0.25 T_{S}+0.2\right) \times 0.2$ \\
\hline
\end{tabular}

Table 2: Non-dimensional frequencies of vibration for simply supported isotropic plate with aspect ratio, $a / b=1$ and span to depth ratio, $t / a=0.1 . \bar{\omega}=\omega t \sqrt{\rho / G}$

\begin{tabular}{|c|c|c|c|c|c|c|}
\hline \multicolumn{7}{|l|}{ Theory } \\
\hline Exact & HPSDT [11] & $\begin{array}{l}\text { TSDT } \\
{[12]}\end{array}$ & $\begin{array}{l}\text { HSDT } \\
\text { [13] }\end{array}$ & $\begin{array}{l}\text { FSDT } \\
{[14]}\end{array}$ & $\begin{array}{l}\text { CPT } \\
{[6]}\end{array}$ & $\begin{array}{l}\text { Present study } \\
\text { (Alternative II) }\end{array}$ \\
\hline 0.0932 & 0.0931 & 0.0933 & 0.0931 & 0.0930 & 0.0955 & 0.0942 \\
\hline
\end{tabular}

Table 3: Non-dimensional buckling load factors for simply supported isotropic plates under uniaxial compression

\begin{tabular}{lllll}
\hline \multirow{2}{*}{$a / t$} & Source & Theory & & $\overline{N_{R}}=\frac{N_{R} a^{2}}{\pi^{2} D}$ \\
\hline \multirow{4}{*}{10} & Present & Alternative II & 3.8220 \\
& {$[11]$} & HPSDT & 3.7866 \\
& {$[12]$} & FSDT & 3.7866 \\
& {$[13]$} & HSDT & 3.7865 \\
& {$[6]$} & CPT & 4.0020 \\
\hline \multirow{4}{*}{5} & Present & Alternative II & 3.3682 \\
\hline & {$[11]$} & HPSDT & 3.2653 \\
& {$[12]$} & FSDT & 3.2637 \\
& {$[13]$} & HSDT & 3.2653 \\
\hline
\end{tabular}

Table 4: Non-dimensional buckling load factors for simply supported orthotropic plates under uniaxial compression $v=0.25$

\begin{tabular}{|c|c|c|c|c|c|}
\hline \multirow{3}{*}{$a / t$} & & \multirow{3}{*}{ Theory } & \multicolumn{3}{|c|}{$\overline{N_{R}}=\frac{N_{R} a^{2}}{E_{2} t^{3}}$} \\
\hline & & & \multicolumn{3}{|c|}{$\mathrm{E}_{1} / \mathrm{E}_{2}$} \\
\hline & & & 10 & 25 & 40 \\
\hline & Present & Alternative II & 9.5494 & 16.7831 & 21.8754 \\
\hline & {$[11]$} & HPSDT & 9.2771 & 15.9031 & 20.3841 \\
\hline & [14] & FSDT & 9.2733 & 15.8736 & 20.3044 \\
\hline \multirow[t]{4}{*}{10} & [14] & FSDT & 9.5415 & 16.7699 & 21.8602 \\
\hline & Present & Alternative II & 10.7168 & 21.3583 & 30.8765 \\
\hline & {$[11]$} & HPSDT & 10.6203 & 20.9561 & 30.0252 \\
\hline & [14] & FSDT & 10.6199 & 20.9528 & 30.0139 \\
\hline \multirow[t]{4}{*}{20} & [14] & FSDT & 10.7066 & 21.3363 & 30.8451 \\
\hline & Present & Alternative II & 11.1548 & 23.4267 & 35.6420 \\
\hline & {$[11]$} & HPSDT & 11.1399 & 23.3811 & 35.5538 \\
\hline & [14] & FSDT & 11.1400 & 23.3810 & 35.5538 \\
\hline 100 & [14] & $\mathrm{CPT}$ & 11.1628 & 23.4949 & 35.8307 \\
\hline
\end{tabular}

Table 5: Comparison of fundamental (linear) frequencies of vibration for SSSS and SSFS orthotropic plates

\begin{tabular}{|c|c|c|c|c|c|c|c|}
\hline \multirow[b]{2}{*}{$a / t$} & \multirow[b]{2}{*}{ Source } & \multicolumn{3}{|l|}{ SSSS } & \multicolumn{3}{|l|}{ SSFS } \\
\hline & & $E_{1} / E_{2}=10$ & $E_{1} / E_{2}=25$ & $E_{1} / E_{2}=40$ & $E_{1} / E_{2}=10$ & $E_{1} / E_{2}=25$ & $E_{1} / E_{2}=40$ \\
\hline & Present $(\omega)$ & 0.0110 & 0.0146 & 0.0166 & 0.0098 & 0.0138 & 0.0160 \\
\hline & Present $(\lambda)$ & 0.00175 & 0.00232 & 0.00265 & 0.00156 & 0.00219 & 0.00255 \\
\hline 10 & {$[15](\lambda)$} & 0.00168 & 0.00226 & 0.00253 & 0.00152 & 0.00212 & 0.00245 \\
\hline 20 & Present $(\omega)$ & 0.0058 & 0.0082 & 0.0099 & 0.0052 & 0.0078 & 0.0095 \\
\hline
\end{tabular}


Application of Alternative II Theory to Vibration and Stability ANalysis Of Thick ReCtANGUlar Plates, Ibearugbulem et al.

\begin{tabular}{llllllll}
\hline \multirow{3}{*}{$a / t$} & & SSSS & & & \\
\cline { 2 - 7 } & Source & $E_{1} / E_{2}=10$ & $E_{1} / E_{2}=25$ & $E_{1} / E_{2}=40$ & $E_{1} / E_{2}=10$ & $E_{1} / E_{2}=25$ & $E_{1} / E_{2}=40$ \\
\cline { 2 - 8 } & Present $(\lambda)$ & 0.00093 & 0.00131 & 0.00157 & 0.00082 & 0.00124 & 0.00152 \\
\cline { 2 - 8 } & {$[15](\lambda)$} & 0.00091 & 0.00129 & 0.00154 & 0.00082 & 0.00123 & 0.00150 \\
\hline \multirow{3}{*}{100} & Present $(\omega)$ & 0.0012 & 0.0017 & 0.0021 & 0.0011 & 0.0016 & 0.0021 \\
\cline { 2 - 8 } & Present $(\lambda)$ & 0.00019 & 0.00027 & 0.00034 & 0.00017 & 0.00026 & 0.00033 \\
\cline { 2 - 8 } & {$[15](\lambda)$} & 0.00019 & 0.00027 & 0.00034 & 0.00017 & 0.00026 & 0.00033 \\
\hline
\end{tabular}

\subsection{Solution to the example}

The particular deflection function that satisfies the boundary conditions for SSSS plate [9] is:

$w=A\left(S_{1} R+T_{1} R^{3}+R^{4}\right)\left(S_{1} Q+T_{1} Q^{3}\right.$

$$
\left.+Q^{4}\right) ; \text { Where } S_{1}=1 \text { and } T_{1}=-2
$$

The particular deflection function that satisfies the boundary conditions for SSFS plate [9] is:

$$
\begin{gathered}
w=A\left(S_{1} R+T_{1} R^{3}+R^{4}\right)\left(S_{2} Q+T_{2} Q^{3}+Q^{4}\right) \\
\text { Where: } \\
S_{2}=-\left(\frac{n_{13} n_{22}-n_{23} n_{12}}{n_{11} n_{22}+n_{21} n_{12}}\right) \text { and } \\
T_{2}=-\left(\frac{n_{13} n_{21}+n_{23} n_{11}}{n_{11} n_{22}+n_{21} n_{12}}\right) \\
n_{11}=3 \mu_{21} ; \quad n_{12}=3 \mu_{21}-\frac{1.875}{P^{2}} ; \\
n_{13}=3 \mu_{21}-\frac{3.75}{P^{2}} \\
n_{21}=6-3 \mu_{21} ; \quad n_{22}=9 \mu_{21}-18+\frac{1.875}{P^{2}} ; \\
n_{23}=12 \mu_{21}-24+\frac{7.5}{P^{2}}
\end{gathered}
$$

The stiffness coefficients for SSSS and SSFS plates [9] are presented in Table 1 .

The values from Table 1 were substituted into Equations (41a), (41b) and (41c) then simplified till Equation (57) to obtain present the desired results.

In this case, $\quad E_{0}=E_{2}=1=e_{22}$. Thus,

$$
\begin{array}{r}
e_{11}=E_{1} ; \quad e_{12}=\mu_{21} E_{1} ; \quad e_{33} \\
=\left(1-\mu_{12} \mu_{21}\right) G_{12} \\
e_{44}=\left(1-\mu_{12} \mu_{21}\right) G_{13} ; \quad e_{55}=\left(1-\mu_{12} \mu_{21}\right) G_{23} ; \\
D_{0}=\frac{E_{0} t^{3}}{12\left\langle 1-\mu_{12} \mu_{21}\right\rangle} ; \quad \frac{D_{0}}{t^{3}}=\frac{E_{2}}{12\left\langle 1-\mu_{12} \mu_{21}\right\rangle}
\end{array}
$$

The example problem was also solved using HPSDT, HSDT, TSDT, FSDT and CPT.

\section{RESULT AND DISCUSSION}

The results of the various analysis using the various plate theories are presented in Table 2 to Table 5 . Table 2 shows the non-dimensional natural frequency for SSSS plate obtained from the present study (using Equation (52)) and those obtained using the other theories while Table 3 shows the non-dimensional buckling load factors for simply supported isotropic plates under uniaxial compression. The nondimensional buckling load factors for simply supported orthotropic plates under uniaxial compression for $v=0.25$ are shown in Table 4 while Table 5 shows the fundamental (linear) frequencies of vibration for SSSS and SSFS orthotropic plates

\subsection{Discussion of results}

Table 2 shows the comparison of non-dimensional fundamental frequencies of vibration for simply supported isotropic plate with aspect ratio $\mathrm{a} / \mathrm{b}=1$ and $t / a=0.1$. The percentage difference with the exact value is $1.07 \%$. When compared with other shear deformation theories, the maximum percentage difference of $1.29 \%$ is recorded. The difference in the results can be attributed to the non-inclusion of shear deformation parameters and following CPT method by previous researchers. Table 3 shows the comparison of non-dimensional buckling load factors for simply supported isotropic plates under uniaxial compression with a maximum difference of $0.93 \%$ with previous researches. The comparison of nondimensional buckling load factors for simply supported orthotropic plates under uniaxial compression is shown on Table 4. The result, which is obtained using, $\overline{N_{R}}=\frac{N_{R} a^{2}}{E_{2} t^{3}}$ shows values for various span/thickness $(a / t)$ ratio and $E_{1} / E_{2}$. Finally, Table 5 shows the comparison of fundamental linear frequencies of vibration of orthotropic plates (SSSS and SSFS). The validity of numeric values from Abaqus [15] which is based on Mindlin - Reissner theory with approximate element size of 0.07 and present study was well established to be the same when the span to depth ratio is 100 (thin plate) for both SSSS and SSSF plates. Furthermore, the results differ with a maximum percentage difference is $4.74 \%$ when the span to depth ratio is 10 or 20 (Thick plate), This is due to non-inclusion of shear deformation parameters as explained earlier. The results of the present study were compared with the result. 
In conclusion, the study has shown significant difference in percentage with respect to thick plate. This might lead to instability or noticeable vibration of structure, for example early cracks, settlement and buckling of structural elements. This method is simply, non-rigorous and straight forward when compared with the methods used in previous researches

\section{REFERENCES}

[1]. A. R. Saidi, A. Rasouli, and S. Sahraee, "Axisymmetric bending and buckling analysis of thick functionally graded circular plates using unconstrained third-order shear deformation plate theory," Composite Structures, vol. 89, no. 1, pp. 110-119, 2009.

[2]. A. S. Sayyad, "Flexure of thick orthotropic plates by exponential shear deformation theory," Latin American Journal of Solids and Structures, vol. 10, pp. 473-490, 2013.

[3]. A. K. Noor and W. S. Burton, "Assessment of Shear Deformation Theories for Multilayered Composite Plates," Applied Mechanics Reviews, vol. 42, no. 1, p. 1, Jan. 1989.

[4]. M. Ghugal and R. P. Shimpi, "A review of refined shear deformation theories of isotropic and anisotropic laminated plates," Journal of Reinforced Plastics and Composites, pp. 775813, 2002.

[5]. Y. M. Ghugal and A. S. Sayyad, "Stress Analysis of Thick Laminated Plates Using Trigonometric Shear Deformation Theory," International Journal of Applied Mechanics, vol. 05, no. 01, p. 1350003, 2013.

[6]. O. M. Ibearugbulem, Note on Rectangular Plate Analysis. Lambert Academic Publishing, 2016.

[7]. O. M. Ibearugbulem, L. S. Gwarah, and C. N. Ibearugbulem, "Use of Polynomial Shape Function in Shear Deformation Theory for Thick Plate Analysis," IOSR Journal of Engineering
(IOSRJEN) WwW.iosrjen.org ISSN, vol. 06, no. 06, pp. 2250-3021, 2016.

[8]. O. Ibearugbulem, K. Njoku, and U. Eziefula, "Full shear deformation for analysis of thick plate," International Journal of Advances in Mechanical and Civil Engineering, vol. 3, no. 6, pp. 44-49, 2016.

[9]. S. Ebirim, "Application of Refined Plate Theory Alternative II to Vibration and Stability of Thick Rectangular Plates," Unplished Ph.D Thesis, Department of Civil Engineering, Federal University of Technology, Owerri, Imo State, Nigeria, 2018.

[10]. E. Ventsel and T. Krauthammer, Thin Plates and Shells: Theory, Analysis and Application, 10th ed. New York: Marcel Dekker, Inc, 2001.

[11]. Ghugal and M. Pawar, "Buckling and vibration of plates by hyperbolic shear deformation theory," Journal of Aerospace Engineering \& Technology, vol. 1, no. 1, pp. 1-12, 2011.

[12]. I. Shufrin and M. Eisenberger, "Stability and vibration of shear deformable plates - First order and higher order analyses," International Journal of Solids and Structures, vol. 42, no. 34, pp. 1225-1251, 2005.

[13]. J. N. Reddy and N. D. Phan, "Stability and vibration of isotropic, orthotropic and laminated plates according to a higher-order shear deformation theory," Journal of Sound and Vibration, vol. 98, no. 2, pp. 157-170, 1985.

[14]. H.-T. Thai, S.-E. Kim, and J. Lee, "Buckling analysis of plates using two variable refined plate theory," Proceedings of 8th Pacific Structural Steel Conference - Steel Structures in Natural Hazards, PSSC 2007, vol. 2, pp. 455462, 2007.

[15]. Simulia, Abaqus/Standard Theory Manual. K. Hibbitt, Sorenson, Inc./HKS, Pawtucket, Rhode Island, 6.8. 2017. 